OPEN ACCESS

Edited by:

Giovanni Di Bonaventura, Università degli Studi G. d'Annunzio

Chieti e Pescara, Italy

Reviewed by:

Tridib Ganguly,

University of Florida, United States

Elisa Borghi,

University of Milan, Italy

*Correspondence:

Ronald Dixon

rdixon@lincoln.ac.uk

Specialty section: This article was submitted to

Infectious Diseases,

a section of the journal

Frontiers in Microbiology

Received: 20 November 2018

Accepted: 23 May 2019

Published: 03 July 2019

Citation:

Kay S, Edwards J, Brown J and Dixon R (2019) Galleria mellonella

Infection Model Identifies Both High

and Low Lethality of Clostridium perfringens Toxigenic Strains

and Their Response to Antimicrobials.

Front. Microbiol. 10:1281.

doi: 10.3389/fmicb.2019.01281

\section{Galleria mellonella Infection Model Identifies Both High and Low Lethality of Clostridium perfringens Toxigenic Strains and Their Response to Antimicrobials}

\author{
Sammy Kay ${ }^{1,2}$, Joseph Edwards ${ }^{1,2}$, Joseph Brown ${ }^{2}$ and Ronald Dixon ${ }^{\text {* }}$ \\ ${ }^{1}$ School of Life Sciences, Joseph Banks Laboratories, University of Lincoln, Lincoln, United Kingdom, ${ }^{2}$ Arden \\ Biotechnology, Lincoln, United Kingdom
}

Research progress into mechanisms of the anaerobe Clostridium perfringens and associated diseases has been frustrated by the lack of reliable infection models. Wax moth larvae (Galleria mellonella) have emerged as a viable alternative to other models of infection since they are economic, survive at $37^{\circ} \mathrm{C}$ and require no specialist equipment. This study aims to establish to what extent $G$. mellonella larvae can be used to study the virulence of $C$. perfringens strains and its suitability for studying novel treatment strategies by an improved time-lapse approach to data collection. Mortality and morbidity rates of larvae challenged with $10^{5} \mathrm{CFU}$ of $\mathrm{C}$. perfringens isolates from various sources were observed over $72 \mathrm{~h}$ and dose response data obtained. Phenoloxidase enzyme activity was investigated as a marker for immune response and tissue burden assessed by histopathological techniques. Results demonstrate that C. perfringens is pathogenic toward G. mellonella although potency varies dramatically between C. perfringens isolates and the reference strain ATCC 13124 was shown to be avirulent. Infection with $C$. perfringens strains activated the melanisation pathway resulting in melanin deposition but no increase in enzyme activity was observed. Efficacy of antibiotic therapy (penicillin G, bacitracin, neomycin, and tetracycline) administered parenterally to some extent correlates with that of in vitro analysis. The findings suggest G. mellonella might be a useful in vivo model of infection and convenient as a prescreening assay for virulence of $C$. perfringens strains or as a simple, cheap and rapid in vivo assay in the first stage development of novel therapeutics against anaerobes.

\section{HIGHLIGHTS}

- Potential novel in vivo model for the study of Clostridium perfringens infection.

- Novel time-lapse approach to data collection.

- First report of the pathogenicity of C. perfringens toward G. mellonella.

- First report of the efficacy of antibiotic therapy in response to C. perfringens infection in G. mellonella.

Keywords: alternative model, insect model, host-pathogen interactions, infectivity, greater wax moth 


\section{INTRODUCTION}

Insect models have been shown to be helpful in our understanding of the virulence of bacterial pathogens in humans (Tsai et al., 2016). Insects share some similarities with mammalian processes and possess a basic innate immune system (Ramarao et al., 2012). Alternative model organisms such as the zebra fish (Danio rerio), silkworm (Bombyx mori), tobacco hornworm (Manduca sexta), fruit fly (Drosophila melanogaster), and nematodes (Caenorhabditis elegans) (Browne et al., 2013) are not associated with the same ethical considerations as in the use of higher vertebrates (Peterson et al., 2008). The larval stage of the Greater Wax moth (Galleria mellonella) has emerged as an insect model of particular value since it survives at $37^{\circ} \mathrm{C}$, which is essential for demonstration of specific microbial virulence factors (Smoot et al., 2001).

The larvae are inexpensive to obtain and easy to maintain using basic equipment (Ramarao et al., 2012). The model does not require ethical approval and coupled with fast reproduction time, allows for a high-throughput of experiments compared with mammalian systems. Whilst G. mellonella has not evolved an adaptive immune response, they possess a semi-complex cellular and humoral innate immunity. This innate system, in insects, shares remarkable similarities to that of mammals (Jander et al., 2000). The cellular immune response in the larvae consists of haemocytes which play a role in phagocytosis, encapsulation and clotting as an antimicrobial response (Tojo et al., 2000). The humoral response consists of various antimicrobial peptides, opsonins, extracellular nucleic acid traps and the phenol-oxidase pathway as described recently in detail by Tsai et al. (2016).

Development of infection models with G. mellonella larval hosts has involved a diverse range of microbes including: Cryptococcus neoformans (Mylonakis et al., 2005), Burkholderia cepacia (Seed and Dennis, 2009), Yersinia pseudotuberculosis (Champion et al., 2009) Acinetobacter baumannii (Peleg et al., 2009), Campylobacter jejuni (Senior et al., 2011), Candida albicans (Brennan et al., 2002), Legionella pneumophilia, (Harding et al., 2013), Pseudomonas aeruginosa (Beeton et al., 2015), Mycobacterium fortuitum (Entwistle and Coote, 2018), and Vibrio parahaemolyticus (Wagley et al., 2017). In the present study, we have investigated the pathogenicity of C. perfringens in G. mellonella larvae. C. perfringens is a ubiquitous Gram-positive, spore-forming, anaerobic bacilli, classically characterized by four major extracellular toxins: alpha (cpa), beta $(c p b)$, epsilon $(e t x)$, and iota ( $i A)$. Recently necrotic enteritis-like B toxin (netB) and enterotoxin (cpe) has been added to the classification system (Table 1; Rood et al., 2018). The bacteria produce a plethora of non-typing toxins including haemolysin, perfringolysin $\mathrm{O}\left(p f_{0} A\right)$, collagenase (colA) (Songer, 1996), beta2 toxin (cpb2) (Van-Asten et al., 2010), and binary enterotoxin $\mathrm{A}$ and $\mathrm{B}$ (becA and $\operatorname{bec} B$ ) (Yonogi et al., 2014) with differing and elusive roles in the bacterial mode of infectivity.

Clostridium perfringens is one of the leading food borne pathogens in the United Kingdom and worldwide. It is implicated in $80-95 \%$ of reported gas gangrene cases (Titball,
TABLE 1 | Toxin type classification system for C. perfringens.

\begin{tabular}{lcccccc}
\hline Type & Alpha & Beta & Epsilon & lota & Entero-toxin & netB* \\
\hline A & + & - & - & - & - & - \\
B & + & + & + & - & - & - \\
C & + & + & - & & $+/-$ & - \\
D & + & - & + & - & $+/-$ & - \\
E & + & - & - & + & $+/-$ & - \\
F & + & - & - & - & + & - \\
G & + & - & - & - & - & + \\
\hline
\end{tabular}

Isolates are characterized by the production of one, or more, of the now six major toxins: alpha, beta, epsilon, iota, *necrotic enteritis like $B$ and enterotoxin. Alpha toxin is produced by all types of C. perfringens (Rood et al., 2018).

2005) and causes large economic losses in the agricultural industry (Wade et al., 2015). Research into this important bacterial pathogen has been slowed by the lack of standardized disease models for assessing virulence and in vivo susceptibility to antimicrobial drugs. The G. mellonella larvae model of infection is developing as an alternative screening method for economically progressing novel antimicrobial compounds prior to mammalian studies.

To date, no detailed reports of $C$. perfringens infection models with G. mellonella have been published. It should be noted, however, that Champion et al. (2016) highlights an unpublished report suggesting alpha and enterotoxin from C. perfringens lack toxicity against the larvae. The aim of this study was to establish the extent of G. mellonella susceptibility to C. perfringens infection as a basis for further virulence studies. This present study investigates the susceptibility to 15 distinct $C$. perfringens isolates from United Kingdom environmental sources including a standard reference strain and explores the response of the larvae to traditional antibiotic therapy. In addition, we introduce a novel imaging capture and time-lapse methodology designed to increase accuracy whilst easily monitoring larval disease progression.

\section{MATERIALS AND METHODS}

\section{Bacterial Strains and Preparation of Inoculum}

Clostridium perfringens was sourced from our culture collection and isolates selected based on toxin profiles (Table 2; Sim et al., 2015). Cultures were stored in 30\% glycerol (Thermo Fisher Scientific, United Kingdom), 70\% brain heart infusion (BHI) media (Oxoid, United Kingdom) at $-80^{\circ} \mathrm{C}$ and cultured on BHI agar (Oxoid, United Kingdom). Liquid cultures were grown to mid-log phase, in BHI or thioglycollate broth (FTG) (Oxoid, United Kingdom) and incubated at $37^{\circ} \mathrm{C}$ for $5 \mathrm{~h}$. Bacterial inocula were adjusted by absorbance $\left(\mathrm{OD}_{620}\right)$ and $\mathrm{CFU} / \mathrm{mL}$ determined by traditional serial dilution and plating on TSC agar (Oxoid, United Kingdom). Cells in adjusted cultures were pelleted by centrifuging at $3170 \times g$ at room temperature for 2 min (Heraeus Megafuge 8) and washed once with $0.1 \%$ peptone water (Oxoid, United Kingdom). Dilutions 
TABLE 2 | Clostridium perfringens toxin profiles of strains and isolates used within this study.

\begin{tabular}{|c|c|c|c|c|c|c|c|c|c|c|c|c|c|c|c|}
\hline Isolate & Source & cpa & $c p b$ & etx & $i A$ & cpe & netb & $c p b 2$ & pfoA & tpel & colA & becA & becB & cna & Type \\
\hline JBFR014 & Free Range Chicken & + & - & - & - & - & - & - & - & - & - & - & - & - & A \\
\hline ATCC 13124 & Type Strain & + & - & - & - & - & - & - & + & - & + & - & - & - & $A$ \\
\hline LPCP001 & Sheep & + & - & - & - & - & - & - & + & - & + & - & - & - & $A$ \\
\hline LPCP023 & Sheep & + & - & - & - & - & - & - & + & - & + & - & - & - & A \\
\hline LPCP042 & Sheep & + & - & - & - & - & - & - & + & - & + & - & - & - & $A$ \\
\hline EDCB283 & Retail Chicken & + & - & - & - & - & + & + & + & - & + & - & - & - & $G$ \\
\hline EDCB093 & Retail Chicken & + & - & - & - & - & - & - & + & + & + & - & - & - & A \\
\hline JBRC013 & Broiler & + & - & - & - & - & + & - & - & - & + & + & - & - & G \\
\hline JBCNJ58 & Broiler & + & - & - & - & - & + & - & + & - & + & - & - & - & $G$ \\
\hline JBCNCRO3 & Broiler & + & - & - & - & - & + & - & + & + & + & - & - & - & G \\
\hline JBCNC055 & Broiler & + & - & - & - & - & - & + & + & - & + & - & - & - & A \\
\hline JBCND021 & Broiler & + & - & - & - & - & + & + & + & - & + & - & - & - & G \\
\hline JBCNI050 & Broiler & + & - & - & - & - & - & - & - & - & - & - & - & - & A \\
\hline JBCND053 & Broiler & + & - & - & - & - & - & - & + & - & + & - & - & - & A \\
\hline JBCNJ055 & Broiler & + & - & - & - & - & - & + & + & - & + & - & - & - & $A$ \\
\hline
\end{tabular}

were drawn into a $1 \mathrm{~mL}$ syringe fitted with a $30 \mathrm{~g}$ sterile needle (BD plastipak).

\section{Galleria mellonella Challenge Assay}

Larvae were purchased from Live Foods United Kingdom (Rooks Bridge, United Kingdom). Upon arrival, they were stored in wood shavings, at room temperature, in the dark. To avoid sampling biases, larvae with any signs of melanisation or deformity were rejected. Larvae were weighed and only larvae meeting the criteria of $250 \pm 50 \mathrm{mg}$ were utilized. Prior to all injections, larvae were immobilized by standing the 24 micro-well plate (Thermo Fisher Scientific, United Kingdom) on ice for $15 \mathrm{~min}$. An automated syringe pump (KD Scientific, United States) was supplied with the drawn $1 \mathrm{~mL}$ syringes and set to administer $1 \mu \mathrm{L} / \mathrm{s}$ for $10 \mathrm{~s}$ (Figure 2B). A safety restraint device was used, outlined by Dalton et al. (2017) (Figure 2C). To minimize adverse effects, larvae were injected into the right posterior proleg (Figure 2C). A melanisation scoring system adapted from Senior et al. (2011) is shown in Figure 1 and employed throughout. Results were obtained longitudinally with a novel image capture system (Figure 2A). Briefly, the system was constructed with a benchtop incubator, with transparent sides and top (Stuart Scientific SI140). Images were recorded by a Logitech $15 \mathrm{MP}$ camera coupled with time-lapse software (SkystudioPro) (Figure 2D). For each experiment, images of the larvae were recorded every $10 \mathrm{~min}$ for $72 \mathrm{~h}$.

\section{Pathogenicity Screening}

Fifteen $C$. perfringens isolates from poultry, goats and their environments along with ATCC 13124 (Table 2) were prepared for inoculation as described above. Groups of 10 larvae were each challenged with $10 \mu \mathrm{l}$ of $10^{7} \mathrm{CFU} / \mathrm{mL}$ and each experiment included 10 larvae injected with $0.1 \%$ peptone water as controls. Larvae were placed in Petri-dishes lined with greaseproof paper to allow for greater contrast in images. All groups were incubated aerobically at $37^{\circ} \mathrm{C}$ and survival recorded at $72 \mathrm{~h}$ p.i. The experiments were repeated in triplicate and an average survival result recorded.

\section{Dose Dependent Challenge}

In dose dependent challenge studies, larvae were prepared by placing one per well in 24 micro-well plastic plates (Thermo Fisher Scientific, United Kingdom). All larvae were treated identically to ensure injection and isolate continuity throughout. ATCC 13124, JBFR014, and JBCNJ055 were investigated by preparing a dilution series of each isolate and larvae were injected with a dilution of $10 \mu \mathrm{L}$ of washed cultures ranging from approximately $10^{3}$ to $10^{7} \mathrm{CFU} / \mathrm{mL}$. In addition, 24 larvae were injected with $10 \mu \mathrm{L}$ of $0.1 \%$ peptone water as negative controls. All six plates for each isolate were recorded simultaneously with the image recording system described previously. The larvae were incubated for $72 \mathrm{~h}$ at $37^{\circ} \mathrm{C}$. Images were taken every $10 \mathrm{~min}$ for $72 \mathrm{~h}$. Melanisation scores and mortality were recorded at $0,12,24$, $36,48,60$, and $72 \mathrm{~h}$ post injection. All experiments were repeated in triplicate and average scores recorded.

\section{Soluble Virulence Factor Trial}

JBCNJ055, EDCB0283, EDCB093, and JBCND053 (Selection based on distinct toxin profiles or sources) were used in cell free trials. $100 \mu \mathrm{l}$ of overnight culture, of each isolate was inoculated in $5 \mathrm{~mL}$ of FTG (Oxoid, United Kingdom) and incubated at $37^{\circ} \mathrm{C}$ for $5 \mathrm{~h}$. The culture was centrifuged at $3170 \times g$ (Heraeus Megafuge 8 ) for $3 \mathrm{~min}$ and $1 \mathrm{~mL}$ of the supernatant was transferred to a $1.5 \mathrm{~mL}$ microcentrifuge tube (Eppendorf, United Kingdom). The remaining supernatant was removed from the bacterial pellet and discarded. The pellet was resuspended in $3 \mathrm{~mL}$ of sterile FTG (Oxoid, United Kingdom) and $1 \mathrm{~mL}$ of the resuspended culture transferred to a $1.5 \mathrm{~mL}$ microcentrifuge tube. The contents of each microcentrifuge tube were drawn into separate $1 \mathrm{~mL}$ sterile syringes (BD Plastipak) and fitted with a $30 \mathrm{~g}$ needle. Ten larvae were challenged with $10 \mathrm{uL}$ of each inoculate, from each isolate and were placed in sterile Petri-dishes lined with greaseproof paper (for better 


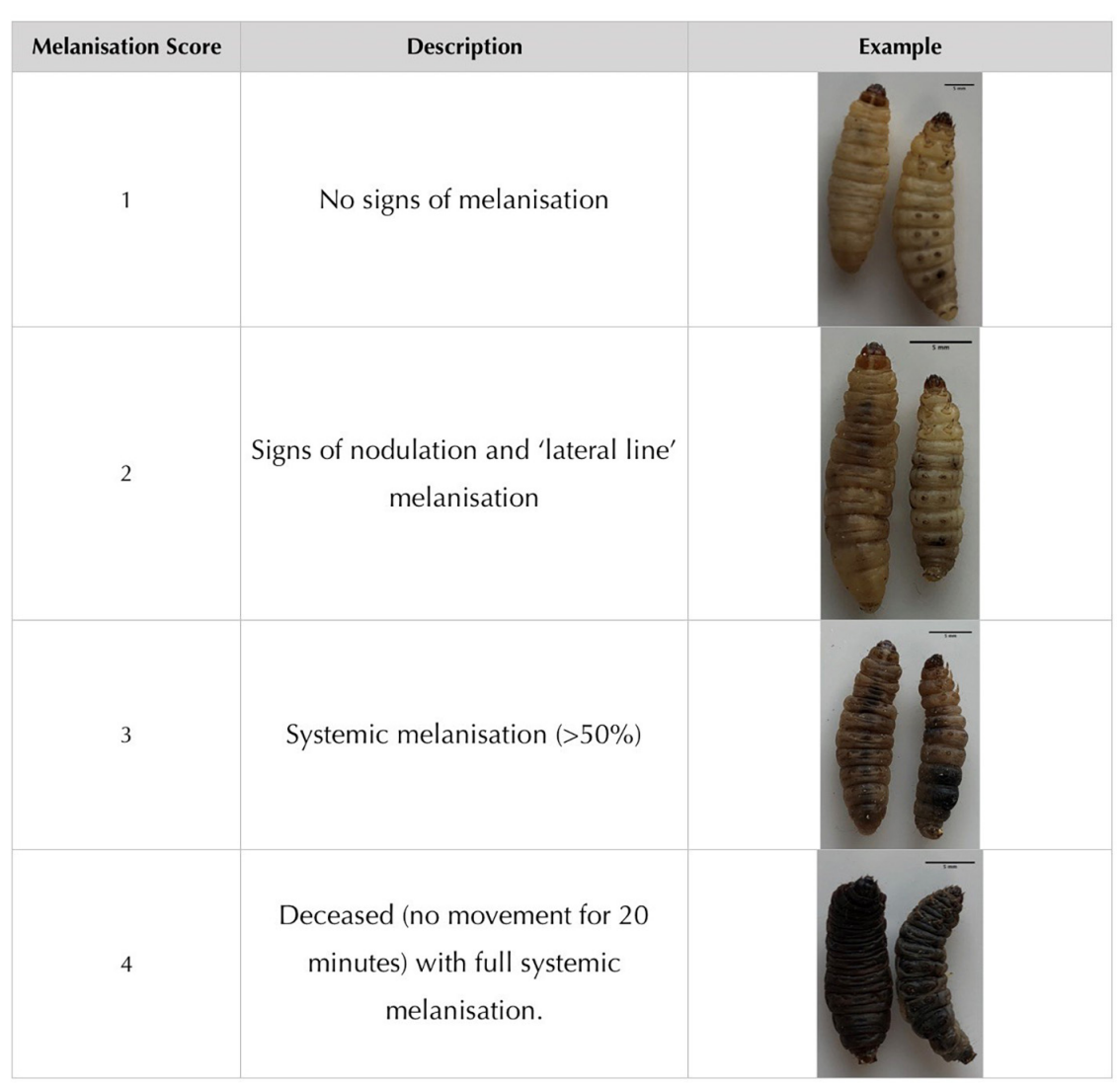

FIGURE 1 | Melanisation scoring system adapted from Senior et al. (2011).

contrast in images). The plates were incubated at $37^{\circ} \mathrm{C}$ for $72 \mathrm{~h}$. Survival percentages were recorded, and each experiment repeated in triplicate.

\section{Isolate Selection for Further Analysis}

Clostridium perfringens JBCNJ055 was selected as a typical recent isolate obtained from a broiler chicken with putative necrotic enteritis in the United Kingdom. Both JBFR014 and JBCNJ055 have been studied extensively in our laboratory and are scheduled to be DNA sequenced. ATCC 13124 was used as a standard reference strain. The remaining isolates used in this study currently have no further characterization.

\section{Haemolymph Extraction}

Twelve larvae were challenged with $10 \mu \mathrm{L}$ of approximately $10^{7} \mathrm{CFU} / \mathrm{mL}$ of $C$. perfringens JBCNJ055 washed culture and 12 larvae injected with $10 \mu \mathrm{L}$ of $0.1 \%$ peptone water as controls. All larvae were incubated at $37^{\circ} \mathrm{C}$ simultaneously for $72 \mathrm{~h}$. At $24 \mathrm{~h}$ intervals post challenge, three larvae from each group were placed into separate sterile $15 \mathrm{~mL}$ centrifuge tubes and submerged in ice for $15 \mathrm{~min}$ to immobilize. Haemolymph was extracted by removing the posterior two segments and bleeding into a sterile pre-chilled $1.5 \mathrm{~mL}$ micro centrifuge tube (Eppendorf, United Kingdom). $25 \mu \mathrm{L}$ of extracted haemolymph was transferred into $75 \mu \mathrm{L}$ of $50 \mathrm{mM}$ PBS (pH 6.5) (Gibco,
United Kingdom) then briefly vortexed and centrifuged to pellet the cells at $11,180 \times g$ for $10 \mathrm{~min}$ at $4^{\circ} \mathrm{C}$. The supernate was transferred into sterile $1.5 \mathrm{~mL}$ tubes. Samples were processed within 15 min of extraction to avoid rapid melanisation.

\section{Phenoloxidase Activity}

Phenoloxidase activity was measured using a microplate enzyme assay (Eleftherianos et al., 2008). Briefly, a reaction mixture containing $115 \mu \mathrm{L}$ of $50 \mathrm{mM}$ PBS (pH 6.5) and $10 \mu \mathrm{L}$ haemolymph plasma was prepared. $25 \mu \mathrm{L}$ of $20 \mathrm{mM} 4$-methyl catechol (Sigma, United Kingdom) was added as enzyme substrate and $2 \mu \mathrm{L}$ of $10 \mathrm{mM}$ Escherichia coli LPS (Sigma, United Kingdom) was added to controls. Plates were subjected to $1 \mathrm{~h}$ of low agitation $(25 \mathrm{rpm})$ at room temperature to activate endogenous pro-phenol-oxidase prior to addition of the substrate. The change in absorbance was read at $490 \mathrm{~nm}$ for $1 \mathrm{~h}$ at room temperature with a reading taken every $60 \mathrm{~s}$ using a plate reader (Fluostar Optima). Each reaction was repeated in triplicate.

\section{Histopathology of Galleria Samples}

Groups of three larvae were injected with $10 \mu \mathrm{L}$ of approximately $10^{7} \mathrm{CFU} / \mathrm{mL}$ of $\mathrm{C}$. perfringens JBCNJ055 and incubated at $37^{\circ} \mathrm{C}$ for $72 \mathrm{~h}$. Control groups were injected with $10 \mu \mathrm{L}$ of $0.1 \%$ peptone water. At $36 \mathrm{~h}$ post injection, larvae were immobilised by 


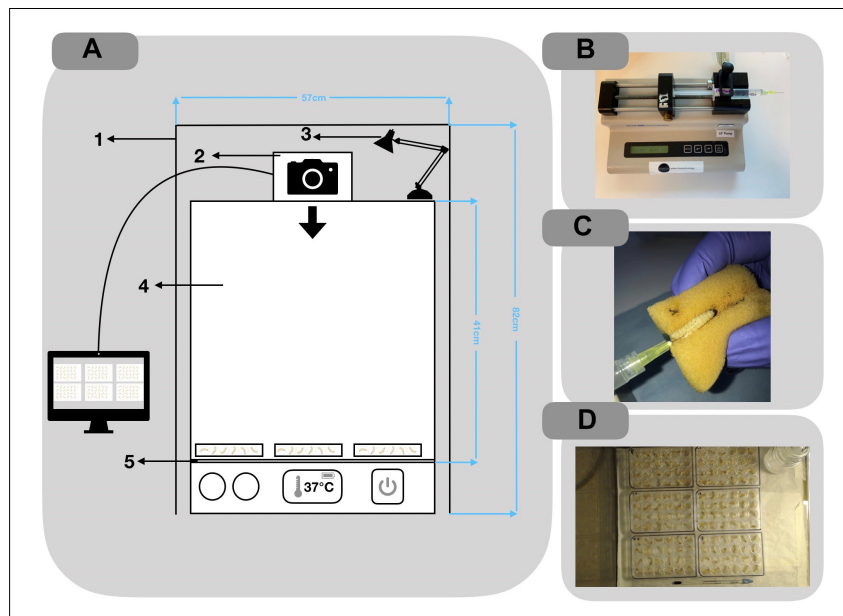

FIGURE 2 | (A) Schematic of the imaging setup used to record time-lapse photography of larvae 1: Black felt shroud to reduce the effects of natural light on quality of images; 2: Camera: Logitek C100 5 mp; 3: table lamp for consistent lighting; 4: perspex incubator: Stuart Scientific SI140; 5: Greaseproof paper for better contrast in images. (B) Automated syringe pump setup used for inoculating larva. (C) Injections were administered through the rear pro-leg. (D) Example of image output from SkyStudio Pro time-lapse software.

submerging in ice for $15 \mathrm{~min}$. Larvae were injected with $150 \mu \mathrm{L}$ of $10 \%$ neutral buffered formalin (Thermo Fisher Scientific, United Kingdom) (for internal fixation) until turgid and stored in $10 \%$ neutral buffered formalin for $36 \mathrm{~h}$ prior to routine tissue processing. Larvae were dissected completely along the sagittal plane, and both halves embedded into wax blocks. Tissue sections (5 $\mu \mathrm{m})$ were cut by routine methods, mounted on glass slides and stained with either $\mathrm{H}+\mathrm{E}$, Masson Fontana, or Gram stains. Sections were imaged using light microscope (Zeiss Primostar) and a 1080 p camera (Mitotic).

\section{MIC Determination}

Clostridium perfringens isolate JBCNJ055 was sub-cultured in $10 \mathrm{~mL}$ of thioglycollate broth (Oxoid, United Kingdom) and incubated for $6 \mathrm{~h}$, aerobically at $37^{\circ} \mathrm{C}$. Broth dilution MIC assays were produced in microtiter plates and prepared with ranges from 128 to $0.25 \mu \mathrm{g} / \mathrm{mL}$ of penicillin $\mathrm{G}$, bacitracin, tetracycline and neomycin. MICs were recorded as the lowest concentration of antimicrobial agent that completely inhibits growth turbidity compared with positive controls where antibiotics were omitted. MIC testing was repeated in triplicate on three occasions.

\section{Antibiotic Therapy}

The same "batch" of larvae (LiveFoods, United Kingdom) were employed for each antibiotic therapy experiment. As positive controls, 24 larvae were challenged with $10 \mu \mathrm{L}$ of approximately $10^{7}$ JBCNJ055 culture followed by an injection of $10 \mu \mathrm{L}$ of sterile $\mathrm{dH}_{2} \mathrm{O}$. A further 24 larvae were injected with $10 \mu \mathrm{L}$ of the same inoculum with a second $10 \mu \mathrm{L}$ injection of either penicillin G (2 mg/kg), bacitracin (64 mg/kg), tetracycline (64 mg/kg), or neomycin (2400 mg/kg); concentrations injected are shown in Table 3. Treatment injections were administered within $15 \mathrm{~min}$ of the bacterial injection. Dosages were chosen to equate with greater than $10 \times$ in vitro MIC. All larvae were subjected to toxicity testing with the maximum doses prior to therapy trials, no melanisation or death was observed. Finally, 24 larvae were injected with $10 \mu \mathrm{L} 0.1 \%$ peptone water as negative controls (Oxoid, United Kingdom). Larvae were incubated simultaneously at $37^{\circ} \mathrm{C}$ for $72 \mathrm{~h}$. Morbidity and mortality scores were recorded at $0,12,24,36,48,60$, and $72 \mathrm{~h}$ post injection. All experiments were repeated in triplicate and average scores recorded.

\section{Statistical Analysis}

The data from each repeat was pooled. Analysis for dose dependent challenges was conducted using Kaplan-Meier survival distributions and tested for statistical significance using the Log rank (Mantel Cox) test (pooled over strata). MannWhitney test was used to highlight statistical significance unless stated otherwise. All tests were carried out in SPSS, 24 (IBM).

\section{RESULTS}

\section{Isolate Pathogenicity}

Figure 3 highlights the variability seen in pathogenicity against $G$. mellonella with different isolates of C. perfringens. The

TABLE 3 | In vitro MIC results with associated therapeutic dosage.

\begin{tabular}{|c|c|c|c|c|c|}
\hline Treatment & $\begin{array}{l}\text { In vitro } \\
\text { MIC } \\
(\mu \mathrm{g} / \mathrm{mL})\end{array}$ & $\begin{array}{c}>\times 10 \\
(\mu \mathrm{g} / \mathrm{mL})\end{array}$ & $\begin{array}{c}\text { Antibiotic } \\
\text { dosage } \times 100 \\
(\mu \mathrm{g} / \mathrm{mL})\end{array}$ & $\begin{array}{c}\text { Final larval } \\
\text { Dosage } \\
\text { ( } \mu \text { g/larva) }\end{array}$ & $\begin{array}{l}\text { Larval survival } \\
\qquad(\% \pm \mathrm{SD})\end{array}$ \\
\hline + Control & & & & 0 & $73.61 \pm 6.36$ \\
\hline - Control & & & & 0 & $100 \pm 0$ \\
\hline Penicillin G & 0.18 & 5 & 500 & 5 & $68.06 \pm 15.77^{*}$ \\
\hline Bacitracin & 1.56 & 15.6 & 1600 & 16 & $94.45 \pm 4.8^{*}$ \\
\hline Neomycin & 60 & 600 & 60,000 & 600 & $65.28 \pm 18.78$ \\
\hline Tetracycline & 1.56 & 15.6 & 1600 & 16 & $79.17 \pm 14.43$ \\
\hline
\end{tabular}

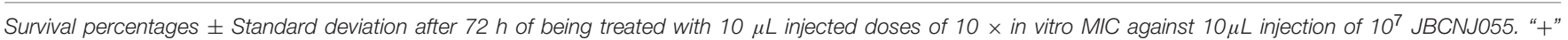

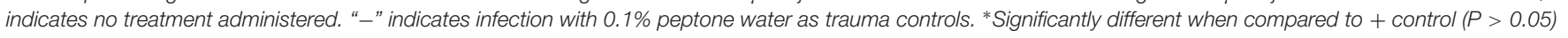
(Man-Whitney U-test). 


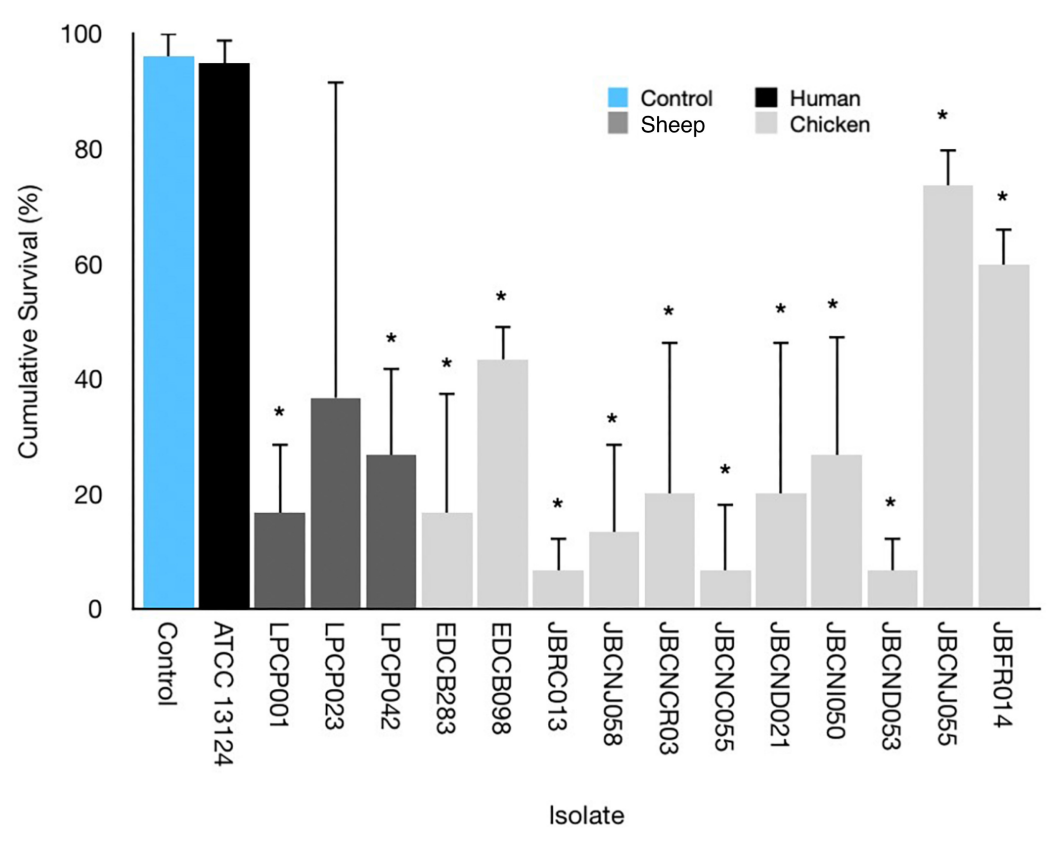

FIGURE 3 | Average survival percentage at $72 \mathrm{~h}$ incubation for G. mellonella infected with $10 \mu \mathrm{L}$ of $10^{7} \mathrm{CFU} / \mathrm{mL}$ of respective isolates. Error bars indicate standard error. Results indicate a variance in lethality of $C$. perfringens infection toward G. mellonella. * $=$ Mann-Whitney $U$-test comparing larval survival to uninfected control.

isolates exhibit a varying degree of pathogenicity with cumulative survival from 5 to $100 \%$.

Parenteral injection of $10^{5}$ CFU C. perfringens JBCNJ055 resulted in disease of the larvae. Larvae which succumb to the infection exhibited nodulation, blackening of the cuticle, and eventually death. Figure 4 shows the rate of development of infection appears dependent on inoculum size as melanisation score increases rapidly with increasing inoculum doses of C. perfringens JBCNJ055. Kaplan-Meier survival distributions for each bacterial inoculum were significant when compared using the log-rank (Mantel-Cox) test (pooled over strata) $(P<0.001)$. Survival probability appears dependent on the number of organisms injected (Figure 5). An inoculum size of $10^{5} \mathrm{CFU} /$ larvae was required for $73.3 \pm 6.66 \%$ survival and induced melanisation $(>3)$ in $>80 \%$ of the population. $10^{4} \mathrm{CFU} /$ larva produced $79.2 \pm 7.2 \%$ survival, however, $<30 \%$ of the population exhibit melanisation scores higher than 2. Further dilutions $\left(10^{3} ; 10^{2} ; 10\right)$ produced $>90 \%$ survival. Injection with $10^{5} \mathrm{CFU} /$ Larva of JBFR014 did incite melanisation in $\sim 40 \%$ of the population, however, survival remained at $>90 \%$. Injection with lower dilutions $\left(10^{3} ; 10^{2} ; 10\right)$ resulted in no visible blackening of the larvae. Interestingly, injection with $10 \mu \mathrm{L}$ of $10^{7} \mathrm{CFU} / \mathrm{mL}$ ATCC 13124 C. perfringens did not cause visible disease to the larvae within the $72 \mathrm{~h}$ window investigated. Melanisation did occur at the site of injection, however, no further immune response was visually observed. Survival remained above $95 \%$ at each inoculum group investigated.

\section{Phenoloxidase}

The results of the phenoloxidase assays shows differences between the control and inoculated groups but not at a significant difference level. Infected groups demonstrated a melanisation score of $3 \pm 0.8$ at $48 \mathrm{~h}$ post inoculation compared to no melanisation in $0.1 \%$ peptone controls.

\section{Cell-Free Toxicity}

All washed harvested cells of $C$. perfringens isolates tested were shown to cause disease in larvae with varying degrees of potency. Furthermore cell-free supernatants of these isolates appeared as potent or demonstrated increased potency compared to uninfected controls of larvae (Figure 6).

\section{Histopathology of Galleria Samples}

Figure 7 shows a distinct response of larvae to $C$. perfringens demonstrating aggregation and nodulation of haemocytes compared to uninfected controls There is clear loss of structure and tissue damage in samples which had been injected with $10^{5}$ CFU/Larva of $C$. perfringens JBCNJ055, with an abundance of melanin demonstrated by Masson Fontana staining compared with negative uninfected controls. Gram-positive cells characteristic of Clostridia were observed associated with tissue remnants in larval sections from C. perfringens infected larvae.

\section{Antibiotic Therapy}

No melanisation or death of larvae was observed with any antibiotics in the absence of infection prior to therapy trials (data not shown). Table 3 shows penicillin $G$ to be the most effective agent in vitro against JBCNJ055 (MIC: $0.18 \mu \mathrm{g} / \mathrm{mL}$ ), however, in vivo larval survival was reduced by $7.5 \%$ compared with controls. Interestingly, Figure 8 shows that the penicillin $G$ treatment in infected larvae resulted in a rapid onset of melanisation compared with controls. We have observed a potent 


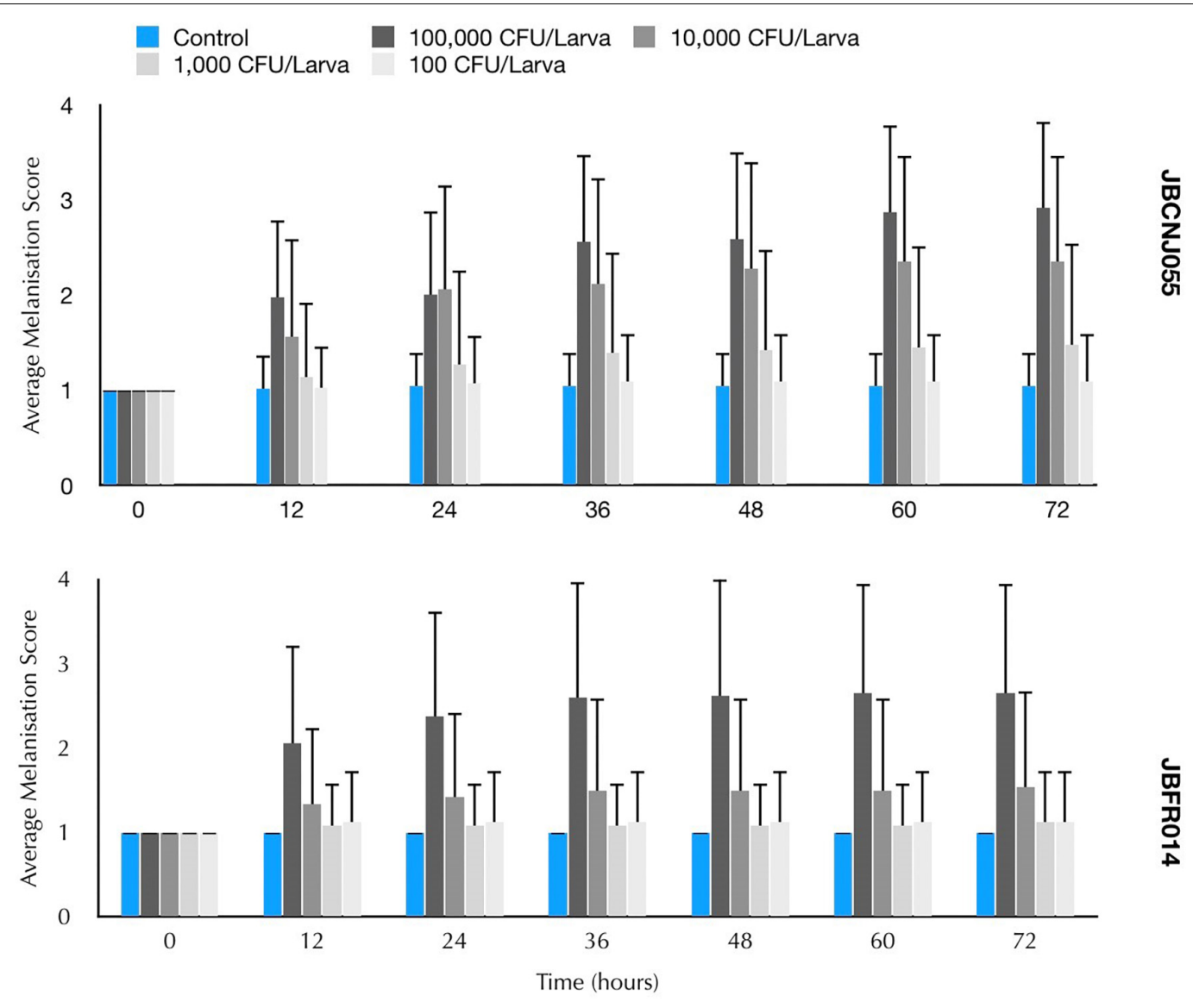

FIGURE 4 | Mean melanisation score of pooled data, $72 \mathrm{~h}$ post inoculation of $10 \mu \mathrm{L}$ of a range of dilutions from $10^{7}$ to $10^{3} \mathrm{CFU} / \mathrm{mL}$ of $\mathrm{Clostridium} \mathrm{perfringens}$ JBCNJ055 and JBFR014. Scoring system is as follows: 1 = healthy, $2=<50 \%$ melanisation, $3=>50 \%$ melanisation, $4=$ dead. Data shown is pooled from three distinct repeats and error bars represent standard deviation. Injection with $10 \mu \mathrm{l}$ of $10^{7}$ was required to cause potent melanisation resulting in scores higher than 3 . Survival percentages of $\mathrm{G}$. mellonella after inoculation with $10 \mu \mathrm{L}$ of a range of dilutions $10^{5}-10 \mathrm{CFU} / \mathrm{mL}$ of $\mathrm{C}$. perfringens.

systemic melanisation in $>80 \%$ of the larvae in under $60 \mathrm{~min}$ after the treatment dose was administered. Injection of penicillin $\mathrm{G}$ alone was not sufficient to cause this potent reaction. Both tetracycline and bacitracin were effective against $C$. perfringens JBCNJ055 in vitro (MIC: $1.56 \mu \mathrm{g} / \mathrm{mL}$ ). In vivo, tetracycline increased larval survival by $7.5 \%$ whereas bacitracin greatly increased larval survival by a remarkable $28.3 \%$ making it the most effective agent in vivo (Table 3). Unsurprisingly, neomycin was the least effective in vitro (MIC: $60 \mu \mathrm{g} / \mathrm{mL}$ ) alongside a reduction in larval survival in vivo of $11.3 \%$.

\section{DISCUSSION}

Galleria mellonella is becoming a well established in vivo model for virulence studies of a number of important microbes and is featuring prominently in the literature as more pathogens are investigated. The advantages of an insect model over mammalian studies in terms of ethics, speed and research cost are becoming apparent with increasing data (Jander et al., 2000). The time lapse features developed here allows for reduced labor inputs although it is not possible to physically manipulate the larvae, a common practice in assessing larval mortality (Peleg et al., 2009; Harding et al., 2013; Beeton et al., 2015). This has been overcome in the present study by classifying dead insects by a melanisation score of 4 (Figure 1) coupled with no movement for $20 \mathrm{~min}$ (2 frames). Time-lapse methodologies, however, increase precision by reducing intermittent $12 \mathrm{~h}$ observations to $10 \mathrm{~min}$ increments. The increase in precision is of benefit when studying pathogens with rapid disease progression. Inherent bias in the manual scoring of the video should be noted but this can be reduced by our current development of an automated melanisation recognition system utilizing the time-lapse feature which will allow for a more robust and standardized scoring system and reduced processing time.

\section{Galleria mellonella and Clostridium perfringens}

Clostridium perfringens is a ubiquitous organism, with a potential arsenal of toxins and hydrolytic enzymes associated with pathogenicity (Petit et al., 1999). The anaerobic pathogen is economically devastating to the global food industry and is 

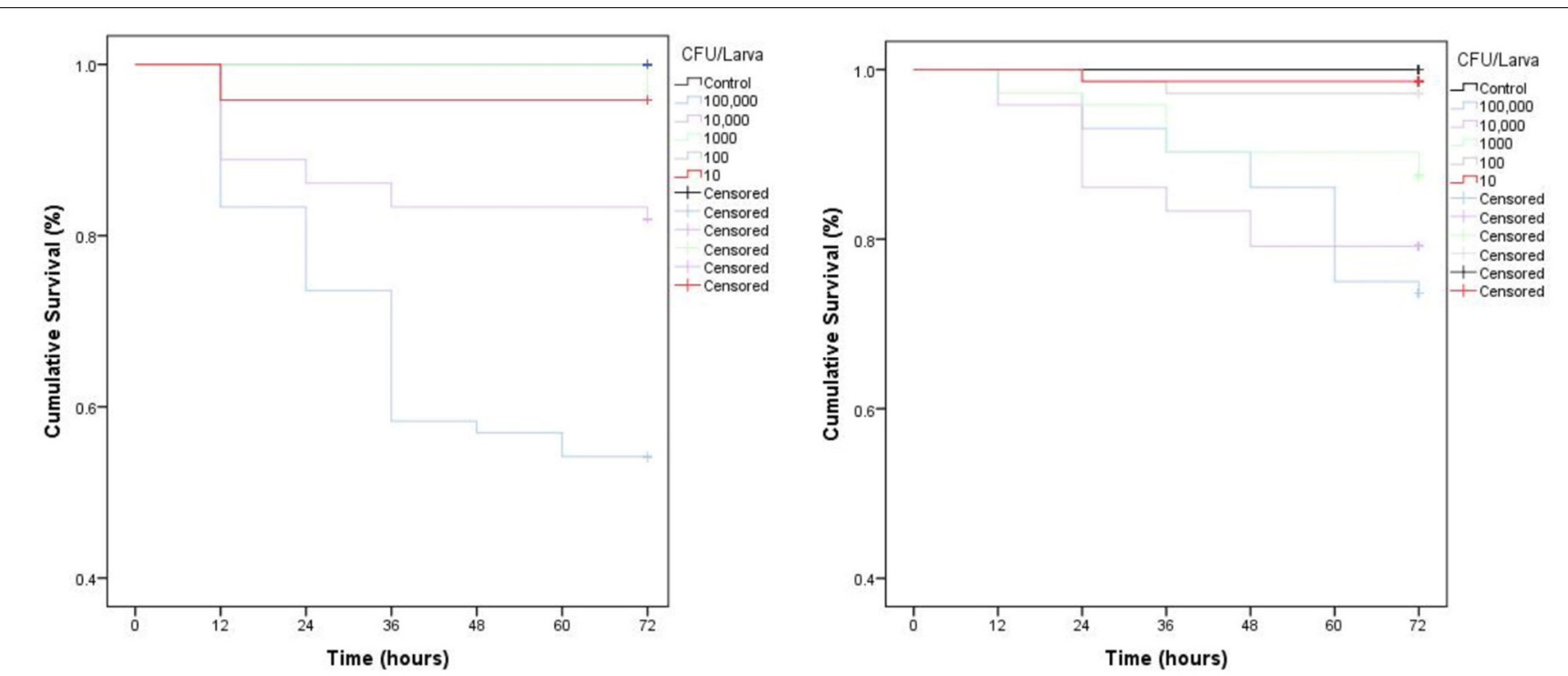

FIGURE 5 | Kaplan-Meier survival distributions for dose dependent challenges of JBCNJ055 and JBFR014. ATCC 13124 is avirulent at all dosage levels. Three repeats of each experiment were pooled. Results were collated as \% survival. Strikethrough indicates censored data (unaffected larvae). All levels of infection data are significant $(P<0.001)$ [Mantel (cox) log rank test] for both isolates investigated indicating larval survival is based upon the number of bacteria injected.

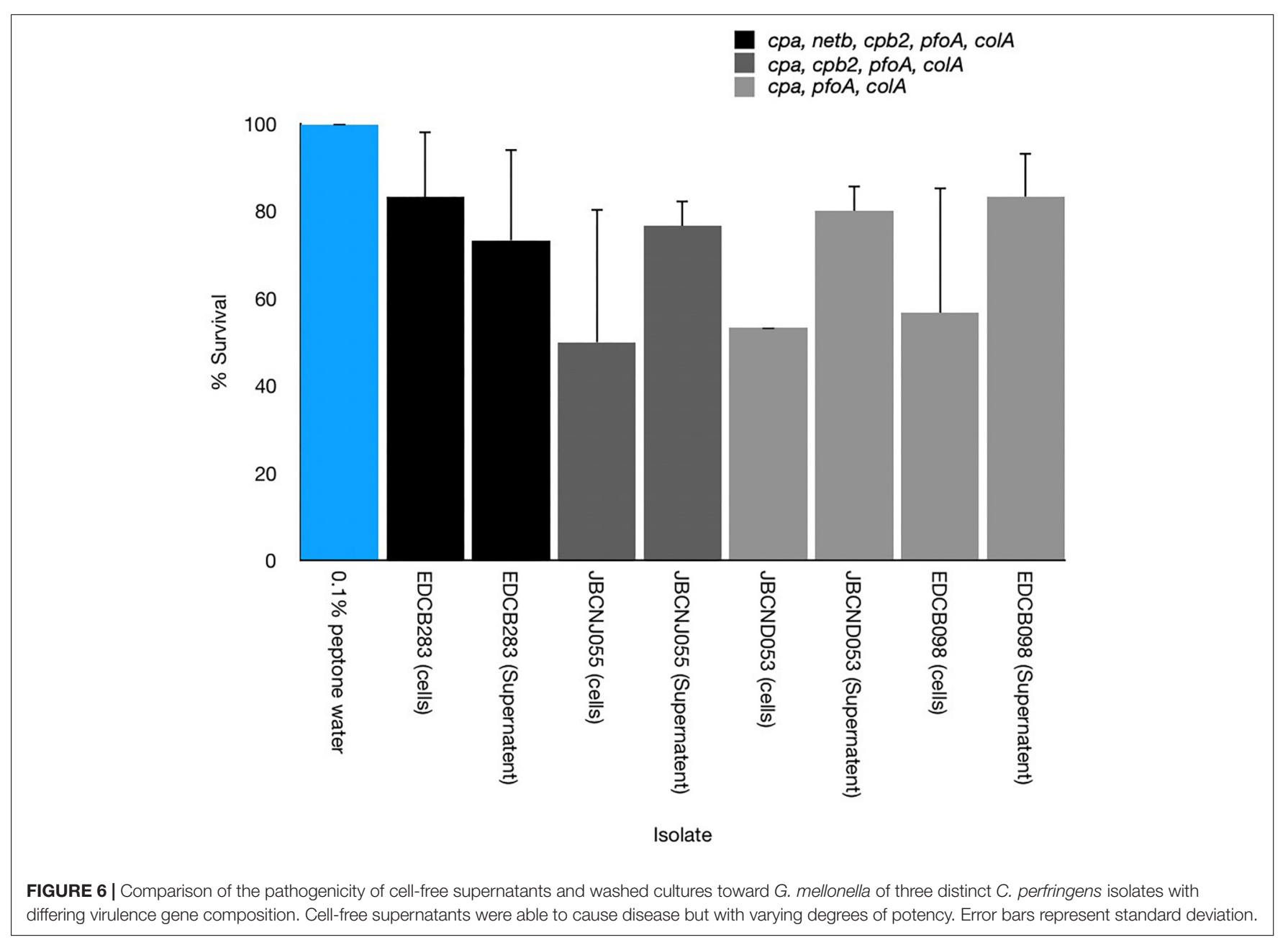




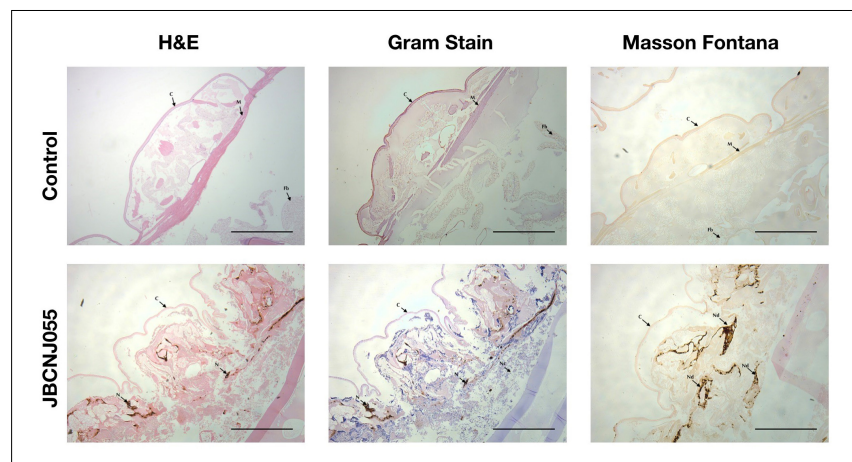

FIGURE 7 | Comparison of G. mellonella histopathology of control and infected groups after $72 \mathrm{~h}$ incubation after inoculation with $10^{5} \mathrm{CFU}$ of JBCNJ055. Sections were stained with $\mathrm{H}+\mathrm{E}$, Gram-stain and Masson Fontana (highlighting melanin deposition). Infected groups show distinct loss of tissue structure, systemic proliferation of Gram-positive bacteria and a large production of melanin within peripheral tissues indicative of systemic pathogenicity. Scale bars represent $1 \mathrm{~mm}$. C, Cuticle; M, Muscle layer; N/Nd, Nodules; Fb, Fat body; Nc, Necrosis.

estimated to cost the poultry sector alone over $\$ 6$ billion (USD) per year (Wade et al., 2015). We have shown for the first time C. perfringens is pathogenic toward G. mellonella larvae although the degree of pathogenicity is distinct between isolates. The differences in pathogenicity of isolates may be due to the differing toxin expression or environmental factors which are thought to play a major role in mammalian CPAD (Borda-Molina et al., 2018). Interestingly we have shown that cell-free supernatants are able to induce disease indicating that soluble factors (toxins) have pathogenic potential compared with negative controls. C. perfringens were able to produce larval death at culture inocula equivalent to other bacteria that have been studied such as C. jejuni ( $10^{6} \mathrm{CFU} /$ Larva) Y. pseudotuberculosis ( $10^{6} \mathrm{CFU} /$ Larva) and C. difficile ( $10^{5} \mathrm{CFU} /$ per larva) (Senior et al., 2011; Champion et al., 2009; Nale et al., 2016), respectively. C. difficile is currently the only clostridial species which has been investigated in detail although $C$. difficle virulence data in the context of the G. mellonella model is limited. An inoculum size of $10^{5} \mathrm{CFU}$ of C. difficile isolate CD105LC2 was sufficient to cause $100 \%$ mortality in larvae (Nale et al., 2016). The variance in lethality between isolates can be seen in other anaerobic enteric pathogens, however, such as campylobacter spp. (Senior et al., 2011), Listeria spp. (Martinez et al., 2017), and some strains of enteric Escherichia spp. (Jønsson et al., 2017). Essential virulence factors in alternative anaerobes such as Listeria monocytogenes have been identified such as the extracellular cytolysin, "listerolysin O" that is essential for virulence, bacterial growth and colonisation of larvae (Joyce and Gahan, 2010).

CPADs are complex and multifactorial processes are still poorly understood (Silva and Lobato, 2015). The use of larval models offers a viable alternative approach for high throughput screening of potentially pathogenic genotypes of anaerobes. In the context of $C$. perfringens, the model would greatly benefit from further validation studies i.e., the investigation of immunological aspects of infection such as haemocyte viability (Bergin et al., 2005), direct toxicological studies and a variety of histopathological approaches (Harding et al., 2013) to further our understanding of pathogenic processes. Investigation of oral inoculation of $C$. perfringens in $G$. mellonella remains unexplored but would offer data on enteric focused pathogenesis and effects on the microbiome (Mukherjee et al., 2013) that is more analogous to that of mammalian clostridial enteritis and therefore a natural further step in the research.

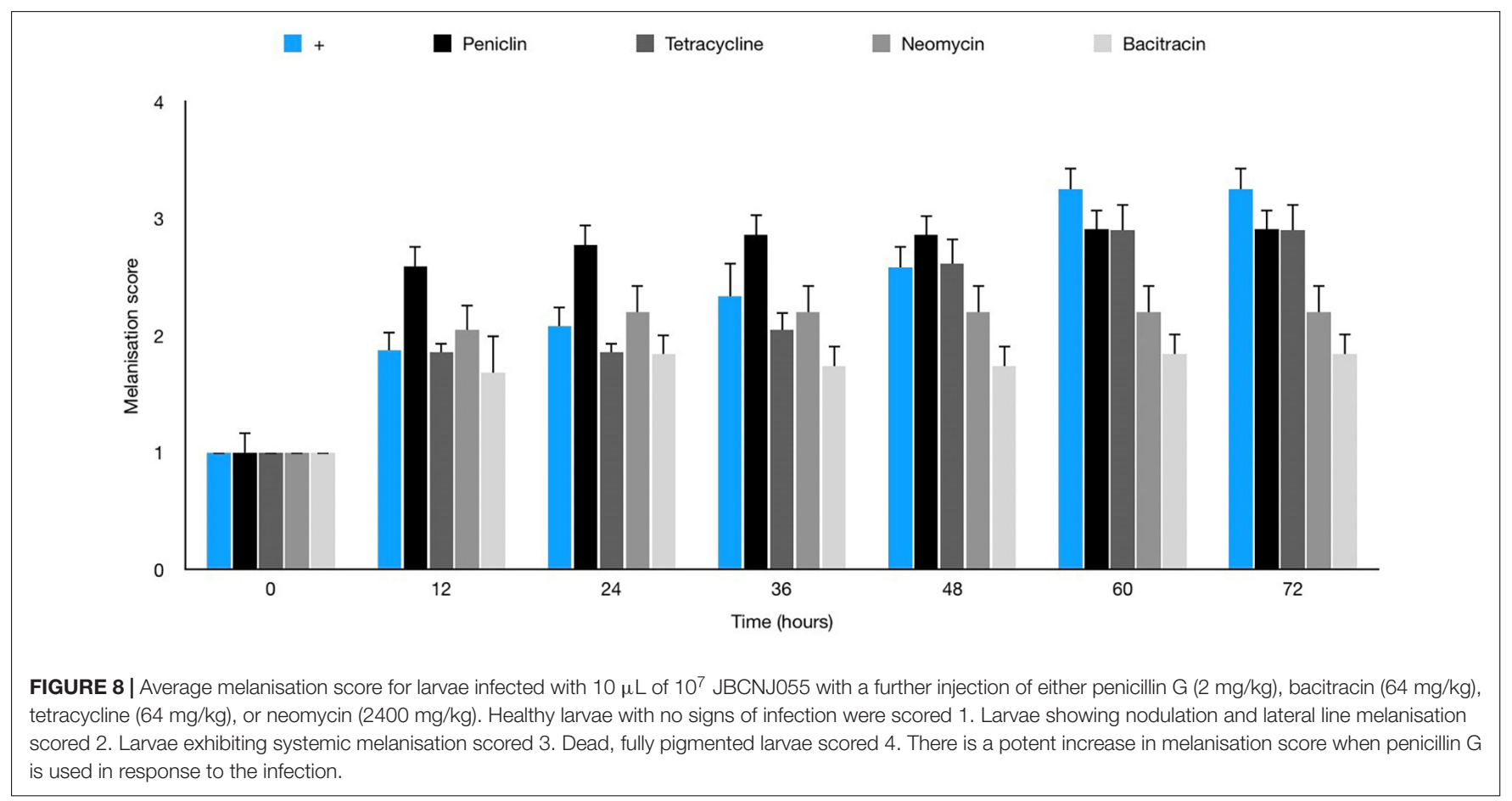


In the present study, C. perfringens activates the phenoloxidase pathway although our enzyme activity data suggests no upregulation of the enzyme in response as seen with other pathogens (Harding et al., 2013). The larvae may allow for an in vivo approach to the further understanding of virulence factors that is challenging in mammals. The potential strength of the model lies with the ability to demonstrate the in vitro virulence diversity. Broader studies are required to draw conclusions about to what extent the mechanism(s) of pathogenicity in larvae relate to mammalian infections.

In our present study, treatment of the C. perfringens infection model with antimicrobials appears to broadly correlate with the in vitro data obtained (Table 3). Penicillin G, interestingly produces a robust and potent reaction by the larvae when administered to $C$. perfringens infected larvae which was not seen when the antibiotic was administered to non-infected larvae. Previous studies (Büyükgüzel and Kalender, 2007) have highlighted that penicillin $G$ induces high levels of oxidative stress in the midgut of G. mellonella which may predispose the larvae to disease and may explain the inefficiency of penicillin in the model which is well known to be highly effective against $C$. perfringens. Tetracycline and bacitracin are both equally effective in vitro against JBCNJ055 but the efficacy of bacitracin in vivo is likely due to its more bactericidal nature against Gram-positive organisms in comparison to the broad spectrum but bacteriostatic tetracycline. The introduction of antifungal compounds to G. mellonella apparently induces independent immune responses potentially masking the efficacy of drugs (Kelly and Kavanagh, 2011). Further research is required here to ensure that antibacterial drug treatments demonstrate no compounding effects on larval immunity. Hence, the model is potentially useful to investigate novel and experimental treatment approaches in the presence of innate immunological

\section{REFERENCES}

Beeton, M. L., Alves, D. R., Enright, M. C., and Jenkins, A. T. (2015). Assessing phage therapy against Pseudomonas aeruginosa using a Galleria mellonella infection model. Int. J. Antimicrob. Agents 46, 196-200. doi: 10.1016/j. ijantimicag.2015.04.005

Bergin, D., Reeves, E. P., Renwick, J., Wientjes, F. B., and Kavanagh, K. (2005). Superoxide production in Galleria mellonella hemocytes: identification of proteins homologous to the nadph oxidase complex of human neutrophils. Infect. Immun. 73, 4161-4170. doi: 10.1128/iai.73.7.4161-4170.2005

Borda-Molina, D., Seifert, J., and Camarinha-Silva, A. (2018). Current perspectives of the chicken gastrointestinal tract and its microbiome. Comput. Struct. Biotechnol. J. 16, 131-139. doi: 10.1016/j.csbj.2018.03.002

Brennan, M., Thomas, D. Y., Whiteway, M., and Kavanagh, K. (2002). Correlation between virulence of Candida albicans mutants in mice and Galleria mellonella larvae. FEMS Immunol. Med. Microbiol. 34, 153-157. doi: 10.1016/s09288244(02)00374-7

Browne, N., Heelan, M., and Kavanagh, K. (2013). An analysis of the structural and functional similarities of insect hemocytes and mammalian phagocytes. Virulence 4, 597-603. doi: 10.4161/viru.25906

Büyükgüzel, E. B., and Kalender, Y. (2007). Penicillin-induced oxidative stress: effects on antioxidative response of midgut tissues in instars of Galleria mellonella. J. Econ. Entomol 100, 1533-1541. doi: 10.1093/jee/100.5.1533

Champion, O. L., Cooper, I. A. M., James, S. L., Ford, D., Karlyshev, A., Wren, B. W., et al. (2009). Galleria mellonella as an alternative infection model for factors without the expense or ethical implications of working with mammals. The data obtained may be used to inform and reduce the number of mammals used for selecting potential lead compounds.

Our methodological advancements described here will improve the ability of the model to evaluate the virulence of pathogens and therapeutic compounds in a more convenient and less laborious way. The data shown in this study suggest that virulence of $C$. perfringens in larvae is distinct and that future studies to dissect the individual contribution of toxin genes in virulence are in hand. We consider that the larvae model will certainly not replace mammalian trials in physiological studies of disease or mechanisms of host response but will be a useful adjunct to potentially characterize unknown pathogenic virulence factors for anaerobic bacteria and perhaps allow pre-screening of antimicrobials against pathogens before embarking on expensive and demanding mammalian studies.

\section{AUTHOR CONTRIBUTIONS}

$\mathrm{RD}$ designed, initiated and overall supervision of project, and critique of the manuscript. JB supported the design of the project and day to day supervision of the laboratory, and supported the preparation of the manuscript. JE undertook partial experimentation and developed the methodology. SK undertook the majority of the experimentation and initial preparation of manuscript.

\section{FUNDING}

This research was supported by the University of Lincoln and Arden Biotechnology Ltd.

Yersinia pseudotuberculosis. Microbiology 155, 1516-1522. doi: 10.1099/mic.0. 026823-0

Champion, O. L., Wagley, S., and Titball, R. W. (2016). Galleria mellonella as a model host for microbiological and toxin research. Virulence 7, 840-845. doi: 10.1080/21505594.2016.1203486

Dalton, J. P., Swift, S., and Wiles, S. (2017). A novel restraint device for injection of Galleria mellonella larvae that minimizes the risk of accidental operator needle stick injury. Front. Cell. Infect. Microbiol. 7:99. doi: 10.3389/fcimb.2017.00099

Eleftherianos, I., Baldwin, H., French-Constant, R. H., and Reynolds, S. E. (2008). Developmental modulation of immunity: changes within the feeding period of the fifth larval stage in the defence reactions of Manduca sexta to infection by Photorhabdus. J. Insect Physiol. 54, 309-318. doi: 10.1016/j.jinsphys.2007. 10.003

Entwistle, M. F., and Coote, P. J. (2018). Evaluation of greater wax moth larvae, Galleria mellonella, as a novel in vivo model for non-tuberculosis mycobacteria infections and antibiotic treatments. J. Med. Microbiol. 677, 585-597. doi: 10.1099/jmm.0.000696

Harding, C. R., Schroeder, G. N., Collins, J. W., and Frankel, G. (2013). Use of Galleria mellonella as a model organism to study Legionella pneumophila infection. J. Vis. Exp. 81:e50964. doi: 10.3791/50964

Jander, G., Rahme, L. G., and Ausubel, F. M. (2000). Positive correlation between virulence of Pseudomonas aeruginosa mutants in mice and insects. J. Bacteriol. 182, 3843-3845. doi: 10.1128/jb.182.13.3843-3845.2000

Jønsson, R., Struve, C., Jenssen, H., and Krogfelt, K. A. (2017). The wax moth Galleria mellonella as a novel model system to study Enteroaggregative 
Escherichia coli pathogenesis. Virulence 8, 1894-1899. doi: 10.1080/21505594. 2016.1256537

Joyce, S. A., and Gahan, C. G. M. (2010). Molecular pathogenesis of Listeria monocytogenes in the alternative model host Galleria mellonella. Microbiology 156, 3456-3468. doi: 10.1099/mic.0.040782-0

Kelly, J., and Kavanagh, K. (2011). Caspofungin primes the immune response of the larvae of Galleria mellonella and induces a non-specific antimicrobial response. J. Med. Microbiol. 60, 189-196. doi: 10.1099/jmm.0.025494-0

Martinez, M. R., Wiedmann, M., Ferguson, M., and Datta, A. R. (2017). Assessment of Listeria monocytogenes virulence in the Galleria mellonella insect larvae model. PLoS One 12:e0184557. doi: 10.1371/journal.pone.018 4557

Mukherjee, K., Raju, R., Fischer, R., and And Vilcinskas, A. (2013). Galleria mellonella as a model host to study gut microbe homeostasis and brain infection by the human pathogen Listeria monocytogenes. J. Adv. Biochem. Eng. 135, 27-39. doi: 10.1007/10_2013_203

Mylonakis, E., Moreno, R., El Khoury, J. B., Idnurm, A., Heitman, J., Stephen, B., et al. (2005). Galleria mellonella as a model system to study Cryptococcus neoformans pathogenesis. Infect. Immun. 73, 3842-3850. doi: 10.1128/iai.73.7. 3842-3850.2005

Nale, J. Y., Chutia, M., Carr, P., Hickenbotham, P. T., and Clokie, M. R. J. (2016). Get in early"; biofilm and wax moth (Galleria mellonella) models reveal new insights into the therapeutic potential of Clostridium difficile bacteriophages. Front. Microbiol. 7:1383. doi: 10.3389/fmicb.2016.01383

Peleg, A. Y., Jara, S., Monga, D., Eliopoulos, G. M., Moellering, R. C., and Mylonakis, E. (2009). Galleria mellonella as a model system to study Acinetobacter baumannii pathogenesis and therapeutics. Antimicrob. Agents Chemother. 53, 2605-2609. doi: 10.1128/AAC.01533-08

Peterson, R. T., Nass, R., Boyd, W. A., Freedman, J. H., Dong, K., and Narahashi, T. (2008). Use of non-mammalian alternative models for neurotoxicological study. Neurotoxicology 29, 545-554. doi: 10.1016/j.neuro.2008. 04.006

Petit, L., Gilbert, M., and Popoff, M. R. (1999). Clostridium perfringens: toxinotype and genotype. Trends Microbiol. 7, 104-110. doi: 10.1016/s0966-842x(98) 01430-9

Ramarao, N., Nielsen-Leroux, C., and Lereclus, D. (2012). The insect Galleria mellonella as a powerful infection model to investigate bacterial pathogenesis. J. Vis. Exp. 70:e4392. doi: 10.3791/4392

Rood, J. I., Adams, V., Lacey, J., Lyras, D., McClane, B. A., Melville, B., et al. (2018). Expansion of the Clostridium perfringens toxin-based typing scheme. Anaerobe 53, 5-10. doi: 10.1016/j.anaerobe.2018.04.011

Seed, K. D., and Dennis, J. J. (2009). Experimental bacteriophage therapy increases survival of Galleria mellonella larvae infected with clinically relevant strains of the Burkholderia cepacia complex. Antimicrob. Agents Chemother. 53, 2205-2208. doi: 10.1128/AAC.01166-08

Senior, N. J., Bagnall, M. C., Champion, O. L., Reynolds, S. E., La Ragione, R. M., Woodward, M. J., et al. (2011). Galleria mellonella as an infection model for Campylobacter jejunii virulence. J. Med. Microbiol. 60, 661-669.
Silva, R. O. S., and Lobato, F. C. F. (2015). Clostridium perfringens: a review of enteric diseases in dogs, cats and wild animals. Anaerobe 33, 14-17. doi: 10.1016/j.anaerobe.2015.01.006

Sim, K., Shaw, A. G., Randell, P., Cox, M. J., McClure, E. Z., Li, M. S., et al. (2015). Dysbiosis anticipating necrotizing enterocolitis in very premature infants. Clin. Infect. Dis. 60, 389-397. doi: 10.1093/cid/ciu822

Smoot, L. M., Smoot, J. C., Graham, M. R., Somerville, G. A., Sturdevant, D. E., Migliaccio, C. A. L., et al. (2001). Global differential gene expression in response to growth temperature alteration in group A Streptococcus. Proc. Natl. Acad. Sci. U.S.A. 98, 10416-10421. doi: 10.1073/pnas.191267598

Songer, J. G. (1996). Clostridial enteric diseases of domestic animals. Clin. Microbiol. Rev. 9, 216-234. doi: 10.1128/cmr.9.2.216

Titball, R. W. (2005). Gas gangrene: an open and closed case. Microbiology 151, 2821-2828. doi: 10.1099/mic.0.28248-0

Tojo, S., Naganuma, F., Arakawa, K., and Yokoo, S. (2000). Involvement of both granular cells and plasmatocytes in phagocytic reactions in the greater wax moth, Galleria mellonella. J. Insect Physiol. 46, 1129-1135. doi: 10.1016/s00221910(99)00223-1

Tsai, C., Loh, J. M. S., and Proft, T. (2016). Galleria mellonella infection models for the study of bacterial diseases and for antimicrobial drug testing. Virulence 7 , 214-299. doi: 10.1080/21505594.2015.1135289

Van-Asten, A. J., Nikolaou, G. N., and Gröne, A. (2010). The occurrence of cpb2toxigenic Clostridium perfringens and the possible role of the cpb2-toxin in enteric disease of domestic animals, wild animals and humans. Vet. J. 183, 135-140. doi: 10.1016/j.tvjl.2008.11.005

Wade, B., Keyburn, A. L., Seemann, T., Rood, J. I., and Moore, R. J. (2015). Binding of Clostridium perfringens to collagen correlates with the ability to cause necrotic enteritis in chickens. Vet. Microbiol. 180, 299-303. doi: 10.1016/ j.vetmic.2015.09.019

Wagley, S., Borne, R., Harrison, J., Baker-Austin, C., Ottaviani, D., Leoni, F., et al. (2017). Galleria mellonella as an infection model to investigate virulence of Vibrio parahaemolyticus. Virulence 9, 197-207. doi: 10.1080/21505594.2017. 1384895

Yonogi, S., Matsuda, S., Kawai, T., Yoda, T., Harada, T., Kumeda, Y., et al. (2014). BEC, a novel enterotoxin of Clostridium perfringens found in human clinical isolates from acute gastroenteritis outbreaks. Infect. Immun. 82, 2390-2399. doi: 10.1128/IAI.01759-14

Conflict of Interest Statement: The authors declare that the research was conducted in the absence of any commercial or financial relationships that could be construed as a potential conflict of interest.

Copyright (C) 2019 Kay, Edwards, Brown and Dixon. This is an open-access article distributed under the terms of the Creative Commons Attribution License (CC BY). The use, distribution or reproduction in other forums is permitted, provided the original author(s) and the copyright owner(s) are credited and that the original publication in this journal is cited, in accordance with accepted academic practice. No use, distribution or reproduction is permitted which does not comply with these terms. 